\title{
The Effect of the Nonlinear Frequency Compression Methods on the Recognition of Monosyllabic Words in Persian Language
}

\author{
Noushin Zarza ${ }^{1}$, Nariman Rahbar ${ }^{2}$, Hassan Haddadzadeh Niri ${ }^{3}$ \\ 1. MSc Student, Department of Audiology, School of Rehabilitation Sciences, Iran University of Medical Science, Tehran, \\ Iran \\ 2. PhD, Department of Audiology, School of Rehabilitation Sciences, Iran University of Medical Science, Tehran, Iran \\ 3. PhD Candidate, Department of Audiology, School of Rehabilitation Sciences, Iran University of Medical Science, \\ Tehran, Iran
}

\begin{tabular}{|c|}
\hline Article Info \\
\hline $\begin{array}{c}\text { Received:2018/11/18; } \\
\text { Accepted:2019/03/17; } \\
\text { Published Online: 2019/03/20 }\end{array}$ \\
\hline DOI: 10.30699/fdisj.2.1.46 \\
\hline How to Cite This Article \\
\hline $\begin{array}{l}\text { Zarza, N., Rahbar, N., Hadd- } \\
\text { adzadeh Niri, H. The Effect } \\
\text { of the Nonlinear Frequency } \\
\text { Compression Methods on the } \\
\text { Recognition of Monosyllabic } \\
\text { Words in Persian Language } \\
\text { Function and Disability Jou } \\
\text { nal. } 2019 \text { (Winter). Vol:2. } \\
\text { No:1. pages:46-52 }\end{array}$ \\
\hline
\end{tabular}

Use your device to scan and read the article online

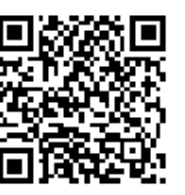

\section{ABSTRACT}

Background and Objectives: The aim of all Frequency Lowering methods is access to high frequencies (HFs) for people with hearing loss. The advantage of these methods has been approved for improving speech perception and limitations reduction of affected people. One of these method is Nonlinear Frequency Compression that reduces the bandwidth of frequency band. This study was carried out to compare the Adaptive Nonlinear Frequency Compression (ANFC) with original algorithm of Nonlinear Frequency Compression (NFC) and Conventional Processing (CP).

Materials and Methods: Thirty people in the range of 18-40 years old with skisloping hearing loss were evaluated. The presence of cochlear dead region at least in one of the frequencies of 1500,2000, 3000 and $4000 \mathrm{~Hz}$ was proved in all cases by performing the Threshold Equalizing Noise test (TEN (HL)). The evaluation was carried out using monosyllabic balanced-word lists in Persian language. Each of Lists used for recognition in one of the three processing modes such as ANFC, NFC and CP which it has been performed in the free field at 90 centimeters distance of the speaker and $0^{\circ}$ azimuth.

Results: The scores of frequency compression algorithms are better than $\mathrm{CP}$ and it leads to improve recognition whereas the scores of NFC and ANFC were similar. In addition, it is not dependent to gender, the obtained scores in three processing modes.

Conclusion: It is more useful for a person with ski-sloping hearing loss to use hearing aids with frequency compression technology rather than CP. In fact, frequency compression gives them a better speech perception, but the effect of the two different algorithms is similar.

Keywords: Nonlinear frequency compression; Hearing loss; Hearing aids; Speech perception

Nariman Rahbar, Department of Audiology, School of Rehabilitation Sciences, Iran University of Medica Science, Tehran, Iran E mail: narimanrahbar@yahoo.com

Copyright (C) 2019, Function and Disability Journal. This is an original open-access article distributed under the terms of the Creative Commons Attribution-noncommercial 4.0 International License which permits copy and redistribution of the material just in noncommercial usages with proper citation.

\section{Introduction}

A person with a ski-sloping hearing loss receives High Frequency (HF) sounds with difficulty due to auditory system impairment. This problem leads to disrupt of receiving some consonants, the discrimination between HF sounds, and the reduction of speech clarity in noisy environments. In addition, if a per- son strikes to hearing loss at childhood, the delay in language and speech development and grammatical problems may occur (Kortekaas \& Stelmachowicz, 2000; McDermott, 2010; Moeller et al., 2007; B. C. Moore, Huss, Vickers, Glasberg, \& Alcantara, 2000; Stelmachowicz, Pittman, Hoover, \& Lewis, 2002; Stelmachowicz, Pittman, Hoover, Lewis, \& Moeller, 2004). The appearance of these problems has an un- 
desirable effect on speech perception, training, social relationships and lifestyle (Hogan \& Turner, 1998), however the use of hearing aids is a good way to prevent or compensate for these problems (Goedegebure, 2005). According to the studies, conventional processing had not satisfied patients with severe to profound hearing loss at HFs (Andrea Simpson, 2009). Physiologically, the most likely cause of this failure is the presence of a cochlear dead region. In this area, the inner hair cell (IHC) is inoperative and does not lead to effective action potential (Brian CJ Moore, 2004). Using frequency lowering (FL) technology in hearing aids is a suitable way to gain the needs of these patients. FL technology includes various methods. These methods transmit audio information from areas of low-performance of cochlea (HFs) to healthy areas (low and mid frequencies) (Braida et al., 1979; Rahbar, 2017; Andrea Simpson, 2009).

Frequency compression is one of the FL methods that includes two main linear and nonlinear types. The linear frequency compression keeps all the frequency spectrum and transmits HF information to lower frequencies while maintaining the constant ratio between its components. This type of compression reduces the pitch of sound and causes abnormal mode, so it was never commercially used in the hearing aid (Andrea Simpson, 2009). In the nonlinear type of frequency compression, the cut-off frequency (CF) is used to improve the audibility of HF sounds, which is based on the audiogram of user's hearing aid (Alnahwi \& AlQudehy, 2015; D Glista \& McDermott, 2008). The $\mathrm{CF}$ is the beginning point of frequency compression; the higher frequency range than $\mathrm{CF}$ is compressed in the lower frequency range, furthermore the input of the hearing aid with reducing spectral details and decreasing in frequency will exist in the output sound of it. In this type of frequency compression, the harmonic ratios change in speech frequency elements, but the sound quality remains close to normal sound (D Glista \& McDermott, 2008; Mueller, Alexander, \& Scollie, 2013; Rahbar, 2017) . Various studies showed that some advantages such as improving the threshold of detection and discrimination of highfrequency consonants, leads to improvement in the identification and comprehension of words, the better place of articulation and voicing; it also improves recognition of monosyllabic words, consonants and sentences in the presence of background noise (Danielle Glista, Scollie, \& Sulkers, 2012; Hillock-Dunn, Buss, Duncan, Roush, \& Leibold, 2014; Kopun et al., 2012; Marchesin \& Iório, 2015; McDermott, 2010; Nyffeler, 2010; Andrea Simpson, 2009; A. Simpson, Hersbach, \& McDermott, 2005).

In 2016 to upgrade the technology, the SoundRecover 2 was introduced with an adaptive $\mathrm{CF}$ feature. Theoretically, in this algorithm, it is possible to maintain more audibility for audiences because one of the two CFs is activated in the moment based on the input frequency audio content. Therefore, if a significant part of the input sound contains low frequencies, the upper CF (higher frequency) is activated; whereas, on the contrary, the lower CF is activated. In the old algorithm, the CF is at least $1500 \mathrm{~Hz}$, while in this algorithm it may be lower than $1500 \mathrm{~Hz}$, so it is possible to apply a lower compression ratio. Applying a lower compression ratio leads to less variation and better protection of the input sounds' frequency shape. Therefore, with using this algorithm, speech perception will be easier and even faster than the original algorithm. In addition, the active frequency spectrum of the hearing aid will increase to $11 \mathrm{KHz}$ with the new algorithm (McDermott, 2010; Rahbar, 2017; Rehmann, Jha, \& Allegro Baumann, 2016; Schmitt, Winkler, Boretzki, \& Holube, 2016; Wolfe et al., 2017).

Applying lower compression ratio and creating a wide and frequency spectrum keep the sound close to normal, which is a crucial factor for speech perception of hearing aid users. In order to specify the superiority of the available technologies, in this study, effectiveness of three different frequency compressions $(\mathrm{CP}$, NFC, ANFC) was compared with the recognition of monosyllabic words. To evaluate three methods, the balanced-word in Persian language, were used. The result of this comparison helps the audiologists in prescribing and setting up appropriate hearing aid for person with cochlear dead region. 


\section{Materials and Methods}

This cross-sectional study investigates the effect of three different modes of frequency compression on speech perception. Thirty patients ( 18 females and 12 males) in the range of 18-40 years (mean 36.8 \pm 2.92 years) with ski-sloping hearing loss were evaluated. All of them had no impairment in conductive system, and their hearing loss was sensorineural. In addition, they had no experience of frequency compression technology in their hearing aids. In this process, after completion of consent form, otoscopy, tympanometry, pure tone audiometry (PTA) and speech audiometry were performed. TEN test was performed as presence of cochlear dead region in participant is essential in this study.

Hearing threshold has been evaluated for both of ears at frequencies of 500, 750, 1000, 1500, 2000, 3000 , and 4000 in two silent and masked paradigm. For masked mode, the TEN test's CD which was connected to the audiometer (type AC-40) was played. The masking noise was $10 \mathrm{~dB}$ above the absolute threshold at the same frequency. To achieve masked threshold, noise and tone were offered to test ear contemporaneous. If the difference between the absolute and masked thresholds is more than $10 \mathrm{~dB}$, it shows the presence of the cochlear dead region (B. Moore, 2007) With this method thirty patients with cochlear dead region were selected whose dead region frequency has been specified in Table 1 .

Table.1 shows the dead region frequencies for each participant. Threshold Equalizing Noise Test (TEN) was used to detect the dead region in each ear. The difference more than $10 \mathrm{~dB}$ between the absolute and masked threshold indicates the dead region in the test.
Table1. Participant Dead Region frequency

\begin{tabular}{ccccc}
\hline \multirow{4}{*}{ Participant } & \multicolumn{4}{c}{ Dead region } \\
\cline { 2 - 5 } & 1500 & 2000 & 3000 & 4000 \\
\hline $1-7$ & & & $*$ & $*$ \\
$18-17$ & & $*$ & $*$ & $*$ \\
$23-30$ & $*$ & $*$ & $*$ & $*$ \\
\hline
\end{tabular}

Considering the symmetry of the ears and putting the hearing aids on the right ear, the evaluation results belong to right ear.

The main part of the study was performed by presenting the balanced list of monosyllabic words in Persian language in free field situation at acoustic chamber also words have been presented in Most Comfortable Level (MCL) (Mosleh, 2001). In order to evaluate each person, the hearing aid was set in $\mathrm{CP}$, NFC and finally ANFC and in every mode there was no fine tuning except for loudness in the individual optimal level.

We used special hearing aid which can be set up in three different frequency compressions. For evaluation of speech perception, balanced-word lists in Persian language has been used. Each of the three lists included 25 words and was used for recognition of the words in one of the three processing modes such as adaptive nonlinear frequency compression (ANFC), original algorithm of nonlinear frequency compression (NFC) and conventional processing (CP) which was performed in the free field at 80-90 centimeters distance of the speaker and $0^{\circ}$ azimuth. After the words were presented in described condition, the number of correct words was calculated.

Since speech processing is performed in the left

Table2. Mean and standard deviation for monosyllabic speech stimulator recognition Scores for each frequency processing (ANFC-NFC-CP)

\begin{tabular}{|ccccccccc}
\hline \multirow{2}{*}{ Processing Type } & \multicolumn{2}{c}{ ANFC } & \multicolumn{3}{c}{ NFC } & \multicolumn{2}{c|}{ CP } \\
\hline Female & SD & Mean & SD & Mean & SD & Mean \\
Male & 1.93 & 16.88 & 1.79 & 17.05 & 1.39 & 15.77 \\
Total Participants & 1.97 & 16.58 & 2.60 & 17.41 & 2.46 & 15.33 \\
\hline
\end{tabular}


Table3. Data Distribution Normality, Homogeneity of the Groups Variances and Mauchly's Test of Sphericity

\begin{tabular}{ccccc} 
variable & \multicolumn{2}{c}{ Leven 's test } & & Mauchly 's test \\
& sig & F & sig & W \\
CP & 0.039 & 4.670 & 0.120 & 0.855 \\
NFC & 0.148 & 2.210 & Box 's M & F \\
ANFC & 0.824 & 0.051 & 0.066 & 1.971
\end{tabular}

hemisphere of the brain and transverse neural pathways in the brain stem and audiogram in the two ears are symmetrical, the hearing aid was put on the right ear and left ear was plugged by spongy earplug (3M 1100 NRR 29dB).

Recognition scores for all participants in three modes (ANFC, NFC and CP) were compared using SPSS 21 software.

\section{Results}

A repeated measures ANOVA was completed with different compression modes (ANFC, NFC and CP).

Comparison of recognition speech stimulus's mean score in the triple position shows the mean obtained score with the NFC is more $(\mathrm{P}<0.05)$ than $\mathrm{CP}$ and
ANFC; also the points obtained with the ANFC compression system are higher than CP mode.

Homogeneity assumption of the variance of intragroup scores was evaluated using Levine's test. Considering the $\mathrm{F}$ as Levine's value which is higher than $\alpha=0.05$ therefore the homogeneity of the data variance and the establishment of regression slope were inferred. According to the table, the results of the test are not significant; therefore, sphericity assumption is established and the result of the intra-group tests was used without modifying the degree of freedom.

According to the Table 4, since the value of $\mathrm{F}$ in the recognition of speech stimulus is significant in "effect of test" at the level of $\alpha=0.05,(\varepsilon 2=345 / 0, \mathrm{P}=0.001$,

Table4. Results of the repeated measures ANCOVA

\begin{tabular}{|c|c|c|c|c|c|c|c|}
\hline Index & Source & $\begin{array}{l}\text { Sum of } \\
\text { Squares }\end{array}$ & $\begin{array}{c}\text { Degrees of } \\
\text { freedom }\end{array}$ & $\begin{array}{c}\text { Mean } \\
\text { Square }\end{array}$ & $\mathbf{F}$ & $\begin{array}{c}\text { Level of } \\
\text { significance }\end{array}$ & $\begin{array}{c}\text { Partial Eta } \\
\text { Squared }\end{array}$ \\
\hline \multirow{2}{*}{ Test Effect } & $\begin{array}{l}\text { Sphericity } \\
\text { Assumed }\end{array}$ & 42.893 & 2 & 21.446 & 14.723 & 0.000 & .345 \\
\hline & $\begin{array}{c}\text { Greenhouse- } \\
\text { Geisser }\end{array}$ & 42.893 & 1.746 & 24.566 & 14.723 & 0.000 & .345 \\
\hline \multirow{2}{*}{$\begin{array}{c}\text { Type of Test } \\
\text { Effect }\end{array}$} & $\begin{array}{l}\text { Sphericity } \\
\text { Assumed }\end{array}$ & 2.670 & 2 & 1.335 & 0.917 & 0.406 & .032 \\
\hline & $\begin{array}{c}\text { Greenhouse- } \\
\text { Geisser }\end{array}$ & 2.670 & 1.746 & 1.529 & 0.917 & 0.395 & .032 \\
\hline
\end{tabular}

Table4. Results of the Post Hoc test on the repeated measurement analysis in ANFC-NFC and CP positions

\begin{tabular}{cccccccc}
\multirow{2}{*}{ Variable } & \multicolumn{2}{c}{ CP-NFC } & \multicolumn{2}{c}{ CP-ANFC } & \multicolumn{3}{c}{ NFC-ANFC } \\
\cline { 2 - 7 } & MD & SD & MD & SD & MD & SD \\
\hline Recognition Score & $-1.681^{*}$ & .320 & $-1.181^{*}$ & 0.366 & 0.500 & 0.259
\end{tabular}

$* \mathrm{P}<0.05$ 
$\mathrm{F}=14.272$ ). The Eta value indicates that the effect of different test methods on the recognition of speech stimulus is $34.5 \%$. The results of the Bonferroni post hoc test (Table 5 and Figure 1) showed that the mean scores of the system stage without frequency compressions were lower than the two test stages, and the scores of the stage of the system of compression system having two cutoffs compared to the system have a significant difference. According to the results that were illustrated in Table 4, the value of $F$ in the gender variable with the type of test, such as the value of the test, is not significant at $\alpha=0.05$, with a value ( $P$ $\left.=0.406, \mathrm{~F}_{(2)}=0.917\right)$.

Based on the mean recognition scores of the $\mathrm{CP}$, it is lower than the NFC and ANFC and scores of the NFC-ANFC were not significantly different.

\section{Discussion}

In the present study we compared efficacy of three different Frequency Compression modes (ANFC, NFC and CP) in speech perception by monosyllabic word in Persian language. Based on this evaluation NFC method are more effective than CP for ski-sloping hearing impaired people. This people experienced better and more comfortable speech perception by using NFC and ANFC. These methods prepared better audibility for HFs. So it is better to use them patients with ski-sloping hearing loss. However, there is no significant difference between two methods based on statistical data. Many studies have been conducted about the effect of frequency compression on auditory skills and high frequency reception. In most of them, phoneme stimuli, monosyllabic words and plural words have been used (Simpson et al., 2005 and 2006, Robinson et al. 2007, Auriemmo et al. 2009, Kuk et al. 2009, Glista et al. 2009, Hillock-Dunn et al. 2014, Picou et al. 2015, Alexander 2016). High-frequency consonants such as fricative and affricate may exceed the bandwidth of CP mode whereas these phonemes are more audible by using FL, thus for these stimuli Frequency compression improve the results (McCreery, Venediktov, Coleman, \& Leech, 2012). So the evaluation by sentences is closer to the actual conversation situation and makes it possible to evaluate several components simultaneously (Hochmuth et al., 2012). Previous Studies of monosyllabic stimuli have shown similar results about superiority of NFC, although the conditions for these evaluations were different. Some of these studies, with different conditions: having the acclimatization period (Simpson et al., 2005) and without it (McCreery et al., 2014), in silence (Gifforf et al. 2007) and with background noise (McCreery and his colleagues, 2014)). The results of some studies also indicated the effeteness of this kind of processing (Bentler et al., 2014); it should be noted that type of hearing loss in the participants was differ-

\section{Estimated Marginal Means of Speech Perception}

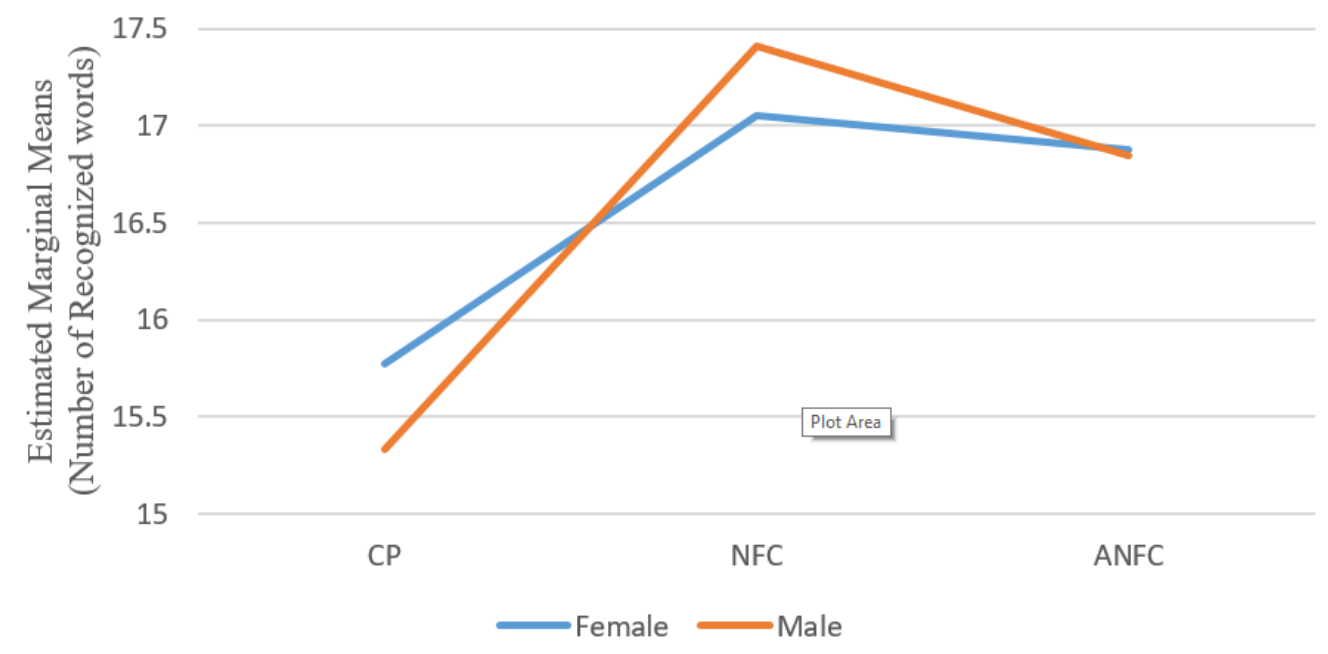

Figure1. Comparison of Recognition Points for monosyllabic spoken stimulus in different locations by gender. 
ent (mild to severe) from the present study.

Based on our results, there was no significant difference between the score obtained with ANFC and NFC modes. Therefore, it can be concluded that frequency compression methods help adult Persian language speakers with symmetrical ski-sloping hearing loss for speech recognition, but the existence of adaptive or static $\mathrm{CF}$ has no difference together. In addition, the scores of recognitions of the speech stimulus of monosyllabic word in the three situations are not different in relation to gender. In the study of Glista et al. (2017) speech perception and word recognition were also evaluated by phoneme perception test (PPT) and there was no difference between the two types of frequency compression in detection and recognition of high frequency consonant; only in the recognition of / $\mathrm{s} /$ centered at $9 \mathrm{KHz}$ (/ s /9) the performance of NFC was better than ANFC. In discrimination of / $\int / 5$ and $/ \mathrm{s} / 6$ the effect of frequency compression methods was similar and only 1 of 8 participants had worse performance by using ANFC (Danielle Glista et al., 2017). Wolfe (2016) and Rehmann (2017) studies results showed the superiority of ANFC than NFC anad CP modes in speech perception. In Rehmann's study were used high frequency consonants and in the Wolfe's study, words and plural names were evaluated. Also, the results have shown the users of the NFC do not require a long period of acclimatization to switch to ANFC(Rehmann et al., 2016; Wolfe et al., 2017).

Most studies that have shown the effect of NFC on auditory skills and speech perception of hearingimpaired people, have also shown efficacy, although the type of speech stimulus (phoneme, monosyllabic word, plural nouns, nonsense word or sentence), study conditions (silent or noisy environment, with or without acclimatization period, evaluation of cochlear dead regions, etc.) and degree of hearing loss of the participant was different. Theoretically, ANFC is more effective than NFC in maintaining sound quality, audibility of HFs, improvement in detection, discrimination and recognition of compressed HF components, acceptable sound quality and fitting for severe-to-profound losses and reduced acclima- tization time. A Few studies and the types of used stimuli do not allow general conclusions (Wolfe et al., 2017). Therefore, it can be concluded that the use of nonlinear frequency compression is due to increased frequency spectrum, hearing improvement, increased sound quality and better speech perception for hearing impaired people; but there was not difference between the effect of nonlinear frequency compression modes (ANFC -NFC) on speech perception.

\section{Acknowledgment}

The authors appreciate cooperation of all participants during this study. We acknowledge all supports of IUMS Deputy of Research.

\section{Conflict of Interest Statement}

Authors declared no conflict of interest.

\section{References}

Alnahwi, M., \& AlQudehy, Z. A. (2015). Comparison between frequency transposition and frequency compression hearing aids. The Egyptian Journal of Otolaryngology, 31(1), 10 .

Braida, L. D., Durlach, N. I., Lippmann, R. P., Hicks, B. L., Rabinowitz, W. M., \& Reed, C. M. (1979). Hearing aids--a review of past research on linear amplification, amplitude compression, and frequency lowering. ASHA monographs(19), 1-114.

Glista, D., Hawkins, M., Bohnert, A., Rehmann, J., Wolfe, J., \& Scollie, S. (2017). The Effect of Adaptive Nonlinear Frequency Compression on Phoneme Perception. American journal of audiology, 26(4), 531-542.

Glista, D., \& McDermott, H. (2008). Phonak SoundRecover: A breakthrough in enhancing intelligibility. Naida Product Information, Switzerland: Phonak Hearing Systems.

Glista, D., Scollie, S., \& Sulkers, J. (2012). Perceptual acclimatization post nonlinear frequency compression hearing aid fitting in older children. Journal of Speech, Language, and Hearing Research, 55(6), 1765-1787.

Goedegebure, A. (2005). Phoneme Compression: processing of the speech signal and effects on speech intelligibility in hearing-Impaired listeners. 
Hillock-Dunn, A., Buss, E., Duncan, N., Roush, P. A., \& Leibold, L. (2014). Effects of nonlinear frequency compression on speech identification in children with hearing loss. Ear and hearing, 35(3), 353.

Hochmuth, S., Brand, T., Zokoll, M. A., Castro, F. Z., Wardenga, N., \& Kollmeier, B. (2012). A Spanish matrix sentence test for assessing speech reception thresholds in noise. International Journal of Audiology, 51(7), 536-544.

Hogan, C. A., \& Turner, C. W. (1998). J Acoust Soc Am, 104(null), 432.

Kopun, J., McCreery, R., Hoover, B., Spalding, J., Brennan, M., \& Stelmachowicz, P. (2012). Effects of exposure on speech recognition with nonlinear frequency compression. Paper presented at the 39th Annual meeting of the American Auditory Society.

Kortekaas, R. W., \& Stelmachowicz, P. G. (2000). Bandwidth effects on children's perception of the inflectional morpheme/s: Acoustical measurements, auditory detection, and clarity rating. Journal of Speech, Language, and Hearing Research, 43(3), 645-660.

Marchesin, V. C., \& Iório, M. C. M. (2015). Study of the long-term effects of frequency compression by behavioral verbal tests in adults. Paper presented at the CoDAS.

McCreery, R. W., Venediktov, R. A., Coleman, J. J., \& Leech, H. M. (2012). An evidence-based systematic review of frequency lowering in hearing aids for school-age children with hearing loss. American journal of audiology.

McDermott, H. (2010). SoundRecover-The importance of wide perceptual bandwidth. Phonak Background Story.

Moeller, M. P., Hoover, B., Putman, C., Arbataitis, K., Bohnenkamp, G., Peterson, B., . . . Stelmachowicz, P. (2007). Vocalizations of infants with hearing loss compared with infants with normal hearing: Part I-phonetic development. Ear and hearing, 28(5), 605-627.

Moore, B. (2007). Prevalence of dead regions in subjects with sensorineural hearing loss. Ear and hearing, 28(2), 231-241.

Moore, B. C. (2004). Dead regions in the cochlea: conceptual foundations, diagnosis, and clinical applications. Ear and hearing, 25(2), 98-116.

Moore, B. C., Huss, M., Vickers, D. A., Glasberg, B. R., \& Alcantara, J. I. (2000). Br J Audiol, 34(null), 205.
Mosleh, M. (2001). Development and evaluation of a speech recognition test for Persian speaking adults. Bimonthly Audiology-Tehran University of Medical Sciences, 9(1), 72-76.

Mueller, H. G., Alexander, J. M., \& Scollie, S. (2013). 20Q: Frequency lowering-The whole shebang. AudiologyOnline, Article, 22679.

Nyffeler, M. (2010). Evidence of improvement in speech intelligibility in noise. Zeitschrift fur Audilogie, 38, 86-95.

Rahbar, N. (2017). Frequency lowering. Auditory and Vestibular Research, 26(1), 4-13.

Rehmann, J., Jha, S., \& Allegro Baumann, S. (2016). SoundRecover2 - The adaptive frequency compression algorithm. Phonak Insight Paper, Phonak, Stäfa, Switzerland. Google Scholar.

Schmitt, N., Winkler, A., Boretzki, M., \& Holube, I. (2016). A phoneme perception test method for high-frequency hearing aid fitting. Journal of the American Academy of Audiology, 27(5), 367-379.

Simpson, A. (2009). Frequency-lowering devices for managing high-frequency hearing loss: A review. Trends in amplification, 13(2), 87-106.

Simpson, A., Hersbach, A. A., \& McDermott, H. J. (2005). Int J Audiol, 44(null), 281.

Stelmachowicz, P. G., Pittman, A. L., Hoover, B. M., \& Lewis, D. E. (2002). Aided perception of/s/and/z/by hearing-impaired children. Ear and hearing, 23(4), 316-324.

Stelmachowicz, P. G., Pittman, A. L., Hoover, B. M., Lewis, D. E., \& Moeller, M. P. (2004). The importance of high-frequency audibility in the speech and language development of children with hearing loss. Archives of Otolaryngology-Head \& Neck Surgery, 130(5), 556-562.

Wolfe, J., Duke, M., Schafer, E. C., Rehmann, J., Jha, S., Allegro Baumann, S., .Jones, C. (2017). Preliminary evaluation of a novel non-linear frequency compression scheme for use in children. International Journal of Audiology, 56(12), 976-988.

Function and Disability Journal 
53. The Effect of the Nonlinear Frequency Compression Methods 